\title{
AUTOMATED ASSEMBLY OF ELECTRONIC DIGITAL MATERIALS
}

\author{
Will Langford \\ Center for Bits and Atoms \\ Massachusetts Institute of \\ Technology \\ Cambridge, MA, USA
}

\author{
Amanda Ghassaei \\ Center for Bits and Atoms \\ Massachusetts Institute of \\ Technology \\ Cambridge, MA, USA
}

\author{
Neil Gershenfeld \\ Center for Bits and Atoms \\ Massachusetts Institute of \\ Technology \\ Cambridge, MA, USA
}

\begin{abstract}
Interest in additive manufacturing has recently been spurred by the promise of multi-material printing and the ability to embed functionality and intelligence into objects. Here, we present an alternative to additive manufacturing, introducing an end-to-end workflow in which discrete building blocks are reversibly joined to produce assemblies called digital materials. We describe the design of the bulk-material building blocks and the devices that are assembled from them. Further, we detail the design and implementation of an automated assembler, which takes advantage of the digital material structure to restore positioning errors within a large tolerance. To generate assembly sequences, we use a novel CAD/CAM workflow for designing, simulating, and assembling digital materials. Finally, we evaluate the structures assembled using this process, showing that the joints perform well under varying conditions and that the assembled structures are functionally precise.
\end{abstract}

\section{INTRODUCTION}

There has been recent interest in fabrication processes and material systems that enable functionality to be embedded within structure. Researchers in the fields of additive manufacturing and digital materials, in particular, have been exploring ways of accomplishing this.

\section{Multi-Material Additive Manufacturing}

The recent interest in additive manufacturing has, in part, been spurred by the promise that complex multi-material structures can be printed with embedded functionality. Recent work has demonstrated that $3 \mathrm{D}$ printing enables the fabrication of ultralight lattices [1], auxetic energy-damping structures [2], and self-folding chains [3]. Commercial 3D printers are able to print objects from a wide range of materials including sintered metals and nylon, UV-cured resin, and thermoplastics like ABS and PLA. However, these printers are generally restricted to printing a single material at a time and only a small number of commercially available printers are able to simultaneously print with multiple materials; those that can, are limited to printing plastics with a relatively narrow range of material properties.

None of these printers, however, have been able match the properties and variety of electronic materials needed to print a full range of electronic devices. While researchers have recently developed conductive ink formulations that enable the controlled deposition of highly-conductive traces $[4,5]$, they have not yet been commercialized (although two are very close to market $[6,7])$. Still, these formulations are one to two orders of magnitude less conductive than bulk metal and often require a post-bake processing step to evaporate the solvent, which limits the substrate material choice.

With these conductive inks, researchers have started to show that it is possible to print functional electronics. Using specially formulated anode and cathode inks, researchers are able to print lithium ion [8] and zinc-air [9] batteries. In another study, the conductive inks were conformally printed on 3D substrates to fabricate efficient antennas [10]. Ink-jet printing has also been used in similar ways to deposit highly conductive silver traces to create electromechanical functionalities like an electrostatic motor [11].

While 3D printers are capable of fabricating objects from a wide range of materials, they all fundamentally perform moreor-less the same task of carefully positioning a print head and depositing or fusing a precise amount of material. If the speed of the positioning is not precisely mapped to the rate of deposition or fusing of material, the fabricated object will have bumps or voids. The accuracy of the final product is therefore ultimately determined by the accuracy of the machine. A model printed on a hobbyist's home 3D printer will come out markedly different from the same model printed on a million dollar commercial 3D printer. 


\section{Digital Material Assembly}

An alternative approach to multi-material additive manufacturing looks to discretize material into individual building blocks. Digital materials are assemblies of individual building blocks, each having a discrete set of possible positions and orientations [12]. The building blocks structurally interlock with neighboring ones such that they register to a lattice. This approach has many traits that make it a compelling alternative to conventional (analog) manufacturing techniques. For one, errors within a tolerance can be restored, enabling an assembler to assemble an object more accurate than itself. That is to say, two very different assemblers, if given the same building blocks, would assemble precisely the same object within the tolerance of the feature size of the building block. Secondly, since the building blocks structurally interlock, dissimilar materials are easily joined, enabling multi-material fabrication with a much broader range of materials. Finally, when the object is no longer needed, the parts can be disassembled and reused rather than thrown away.

A number of different variations of digital materials and methods of discrete assembly have been explored. MacCurdy et al., for example, assembled functional electronic circuits from individual component building blocks [13]. The building blocks themselves were made from printed circuit boards and commercially available press-fit connectors were used to join the blocks to each other. A 3D printer was modified with a part placement head in order to automate the assembly of the blocks. Automated assembly was demonstrated through the assembly of an infrared remote control composed of 17 blocks.

In another work by Hiller et al., a serial voxel assembler was demonstrated, which builds multi-material objects from discrete spherical building blocks [14]. The assembler assembled structures from three different materials simultaneously. The voxels were bound together using a liquid adhesive binder and the researchers showed that structures could be recycled by simply dissolving the binder in water. The assembler was able to place parts at a speed of approximately 2 $\mathrm{Hz}$, which was demonstrated by building a dome shape composed of approximately 400 voxels in three minutes.

In this work, we introduce an end-to-end workflow for discrete assembly, enabling a new kind of electronic material system. This work spans part design and production, digital material CAD/CAM tools, and automated assembly workflows. In comparison with existing approaches, this work uses fundamental building blocks that structurally interlock and shows that complex electronic functionality can be built up from just two bulk material building block-types. Furthermore, we build an automated serial assembler and demonstrate that it can take advantage of the digital material properties to build objects more precise than itself.

\section{DESIGN}

\section{Part Design}

In designing the parts of a digital material system, a number of considerations need to be taken into account, including the level of complexity of the building block. Digital material parts can range in complexity from simple singlematerial spheres [15] to elaborate pre-fabricated modules [16]. In this work, we choose to focus on the low-complexity end of this spectrum, fabricating the building blocks from a single material in a one-step fabrication process.

This design choice impacts many other aspects of the part design. Since the building blocks are made from a single material, they should also be two-dimensional and have a simple geometric shape such that they can be easily fabricated from many different materials at many different length-scales. In this case, a meso-scale block size, pictured in Figure 1, was chosen to balance the difficulty of manipulation and assembly with functional density.

To automate the assembly of the parts, it is desirable to have a build-front, or a single direction from which the parts are inserted. For this reason, the parts in this work have slots on the top and bottom to enable this kind of top-down vertical assembly. This enables parts to connect with neighbors on adjacent layers but not within the same layer. The number of slots (four on each side) is the minimum required to tile the parts orthogonally and maintain the structural integrity of the overall structure. While parts with fewer slots (and connections between layers) are certainly possible, their assemblies require increased design constraints in order to maintain structural integrity. These building blocks have been described in previous work and are referred to as GIK (Great Invention Kit) parts [12].
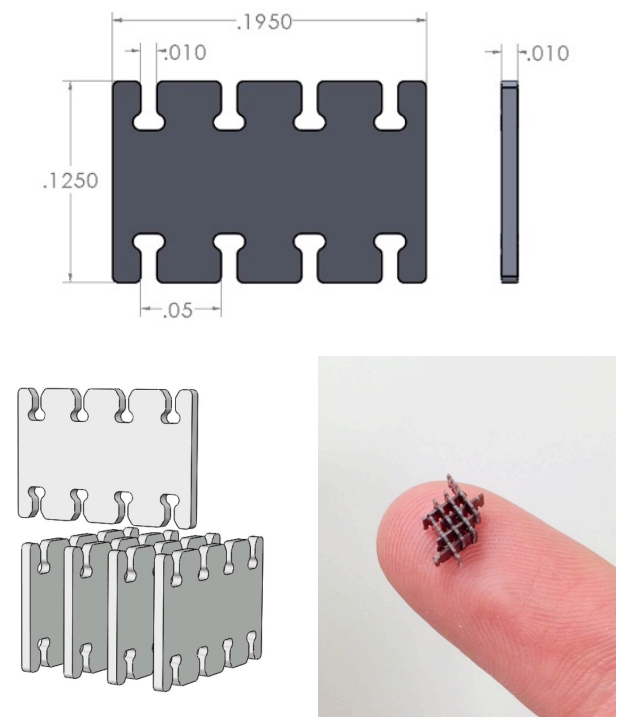

FIGURE 1 - PART GEOMETRY AND ASSEMBLY

[TOP] DIMENSIONED DRAWING OF A GIK PART (ALL DIMENSIONS ARE IN INCHES). [LEFT] ILLUSTRATION SHOWING HOW PARTS ARE JOINED. [RIGHT] AN ASSEMBLY OF GIK PARTS ON A FINGER FOR SCALE. 
A critical part of this work is ensuring a good connection between the individual building blocks. In this case, that means both that the parts structurally interlock, self-aligning with neighboring parts, and that, for electronic assemblies, the conductive parts are joined with little contact resistance. In this work, the slots are sized such that the insertion of one slot into another creates a press-fit joint. The insertion force required for this press-fit is tuned to balance the difficulty of insertion with the strength and reliability of the joint. Experimental evaluation and validation of these joint properties is covered later in Evaluation.

In this work, we fabricate the parts from two different materials: one insulating and one conductive. For the insulating part-type, we use a garolite fiber-epoxy composite, which we use for its electrical resistance, its structural stiffness and strength, as well as its thermal stability. Also, because it is widely used as a substrate for conventional circuit boards, its dielectric properties are well understood. These parts are produced using a custom-built automated desktop punch-press (described in Annex A).

For the conducting part-type, we use brass for its stiffness, hardness, and machinability. Both aluminum and copper parts were tried but proved to be too ductile, deforming rather than creating a strong press-fit joint. While brass is good for prototyping, tin-plated phosphor-bronze alloy may be the ideal material choice for its soft-plating but high strength and stiffness. The conductive parts are produced using a WireEDM; a stack of shim stock is sandwiched between two plates and cut such that 20 parts are made at once.

\section{Device Design}

Using building blocks of this type, it is possible to assemble a number of different functional electronic devices. With just two block-types (conducting and insulating), electronic interconnect can be assembled. With the addition of a third resistive block, it is possible to assemble any passive electronic component. Finally, with the addition of blocks with embedded active electronic functionality (such as diodes and transistors) an entire integrated circuit may be assembled.

This work focuses on assemblies of conducting and insulating block-types and demonstrates the various electronic functionalities that can be assembled from them.

By strategically placing conductive building blocks within a structure, arbitrary electrical traces can be routed, connecting electrical components placed on the surface of the structure. In this case, the pitch of the digital material lattice is conveniently chosen to match the pitch of small outline integrated circuit (SOIC) surface mount components $(1.27 \mathrm{~mm})$. An example of this kind of assembly is pictured in Figure 2 in which conductive and insulating parts are used to replace the conventional traces, pads, vias, and layers of a circuit board with a volume of electronic materials to blink an LED using an ATtiny microcontroller. This three-dimensional "circuit board" was designed and assembled by hand. With the exception of $5 \mathrm{~V}$ power, which was provided externally by the red and black wires, everything required to blink the LED is onboard.

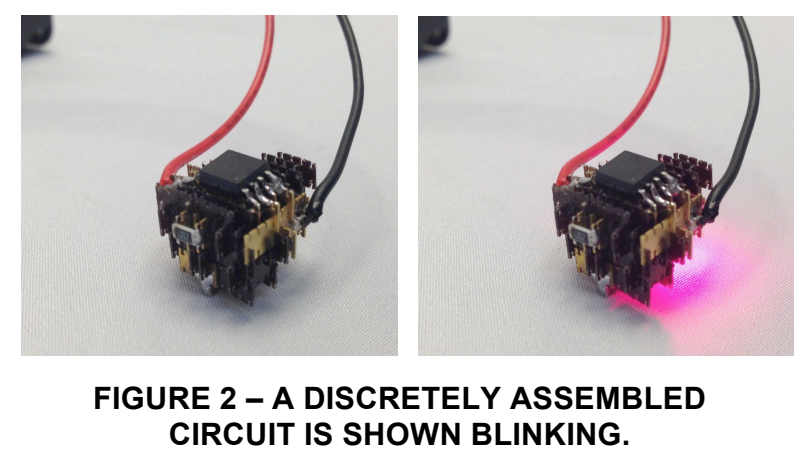

Going beyond circuit boards, by carefully arranging the placement of the conductive blocks in the structure, passive components themselves may be assembled. For example, by placing the conductive blocks in an interdigitated finger arrangement, a capacitor can be assembled. Similarly, by placing the conductive blocks in a spiral or helix we can assemble an inductor.

To demonstrate the assembly of passive components from conductive and insulating blocks, we hand-assembled a capacitor and inductor and characterized their performance as an LC resonator. The capacitor, which measures $19.6 \mathrm{pF}$, is composed of approximately 140 parts and the inductor, which measures $0.44 \mu \mathrm{F}$, is made up of approximately 520 parts. In this example, the resonator is composed of a separate capacitor and inductor, which are wired in series with a short length of wire and hooked up to a signal generator (Agilent E4421B ESG) and oscilloscope (Tektronix MS02024) to measure the frequency response of the combined components. A sine-wave is swept from $500 \mathrm{kHz}$ to $150 \mathrm{MHz}$ and the amplitude and phase of the signal across the capacitor is recorded and plotted in Figure 3 . The quality factor of the resonator is calculated as the ratio of the resonant frequency to the half-power bandwidth and gives a measure of how sharp and resonance zone is. In this case we measure a Q-factor of 4.5 (including a $50 \mathrm{ohm}$ source impedance), which is the same order of magnitude as that of an LC resonator constructed from conventional chip-mount components. 

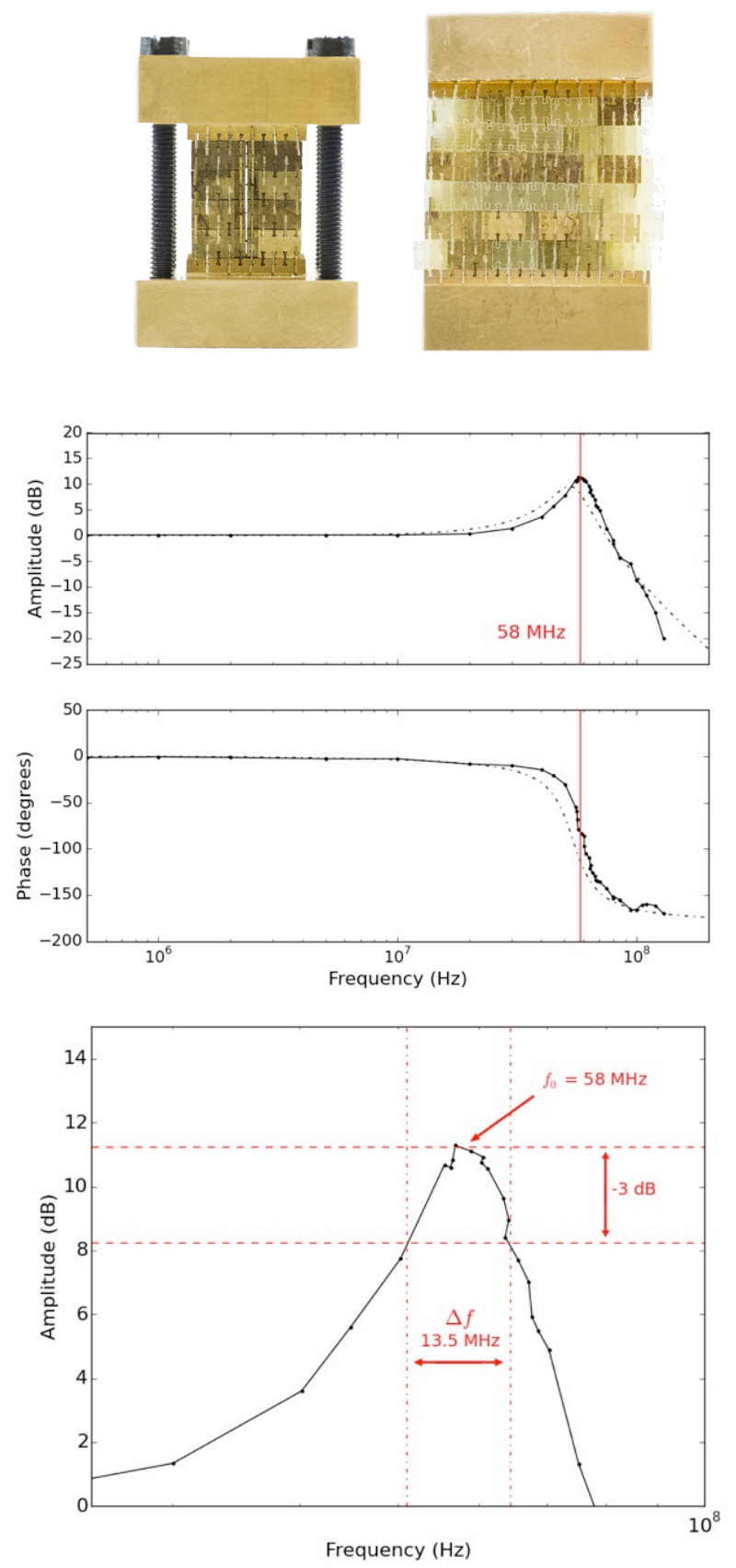

FIGURE 3 - A DISCRETELY ASSEMBLED LC RESONATOR.

[TOP] DISCRETELY ASSEMBLED CAPACITOR (LEFT) AND INDUCTOR (RIGHT). [MIDDLE] FREQUENCY RESPONSE PLOT SHOWING THAT THE COMPONENTS EXHIBIT RESONANCE AT 58MHz. [BOTTOM] ZOOM OF THE RESONANCE PLOT SHOWING THE HALF-POWER BANDWIDTH REGION.

\section{Assembler Design}

These assemblies of hundreds of components took many hours to assemble using the GSWT (graduate student with tweezers) method. One of the primary aims of this work is to detail the design and implementation of an automated means of assembly to increase assembly throughput and allow for greater design complexity.

The design of the assembler in this work resembles that of conventional fabrication machines in many ways. A 4-axis motion gantry is used to position two toolheads spatially with respect to the structure being built. However, the assembler differs from other fabrication tools in that it takes advantage of the inherent qualities of digital material structures. Unlike conventional machine tools, the assembler can correct positioning errors within a tolerance by registering with the digital material lattice, enabling the assembly of structures more accurate than the assembler itself. Similarly, unlike conventional machine tools, an assembler either places a part successfully or it does not. This kind of error is much easier to detect and correct than, for example, depositing thermoplastic slightly too quickly, as might be the case with a fused deposition modeling (FDM) 3D printer.

The assembler presented in this work differs from existing automated assembly machines in a few ways. Unlike the BitBlox assembler [13], which must pick up and place a part, the assembler in this work stores the parts onboard in a magazine, potentially doubling the speed of assembly by eliminating traversals to the part supply area between part placements. This assembler also explicitly takes advantage of the error correction capabilities of the digital materials by adding intentional compliance between the toolhead and the structure to allow the toolhead to register to the digital material lattice. In theory, an assembler may be designed to accept a positioning error up to half of the lattice pitch of the digital material assembly. In this case that would be a positioning error of $0.635 \mathrm{~mm}$.

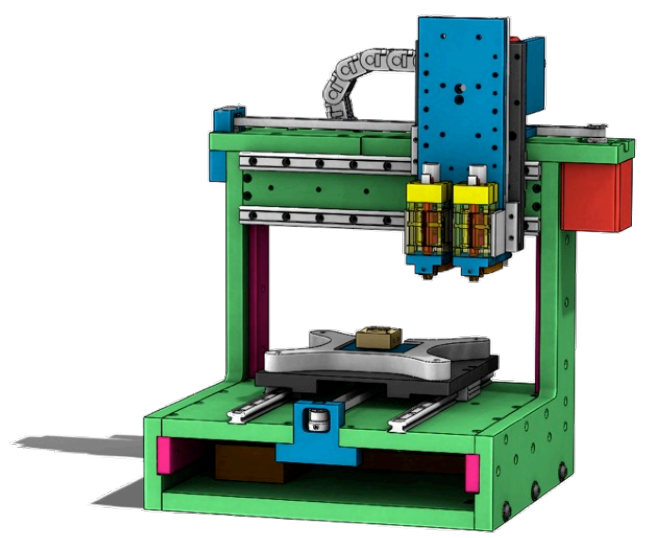

FIGURE 4 - ASSEMBLER DESIGN

\section{IMPLEMENTATION}

This assembler, which we call the "stapler assembler," can be broken down into three primary subsystems: the motion gantry, the part placement or stapler mechanism, and the part storage or magazine. This section will detail their implementation. 


\section{Motion Gantry}

A 4-axis motion gantry is used to position the two toolheads in $\mathrm{X}, \mathrm{Y}, \mathrm{Z}$ and $\mathrm{C}$ (rotation about $\mathrm{Z}$ ) spatial coordinates. The motion gantry is constructed from a steel reinforced HDPE frame. The frame was $\mathrm{CNC}$ milled to precisely position all holes and pockets. Precision ground steel was then bolted to the frame pieces to add rigidity and mass. The axes use polymer linear guideways for a low friction motion in each axis. The axes are powered by high-torque NEMA-17 stepper motors and driven with GT2 timing belts. The motor and power transmission elements were specified to nominally provide a $1 / 16$ th microstep resolution of $5 \mu \mathrm{m}$ and a maximum operating speed of $50 \mathrm{~mm} / \mathrm{s}$ with a potential linear force delivery of $70 \mathrm{~N}$ in each axis.

Some amount of backlash in timing belt transmissions is always present, and needs to be accounted for in order to position precisely. In this case we measured the backlash in the timing belt transmission to be between $125 \mu \mathrm{m}$ and $250 \mu \mathrm{m}$ in the $\mathrm{X}$ - and Y-axes. Fortunately, for our application, it is trivial to compensate for this error by driving the axes so that they always approach a part-placement location from the same direction, effectively nulling the backlash and enabling a positioning repeatability better than $25 \mu \mathrm{m}$ in X-and Y-axes.

To enable the toolhead of the machine to self-align with the digital material structure, we added intentional compliance between build platform and the toolhead through the use of an $\mathrm{XY}$-flexure mechanism located on the Y-axis. The flexural mechanism, which was fabricated by CNC milling, allows deflections of $0.5 \mathrm{~mm}$ to occur with approximately $2 \mathrm{~N}$ of lateral force.

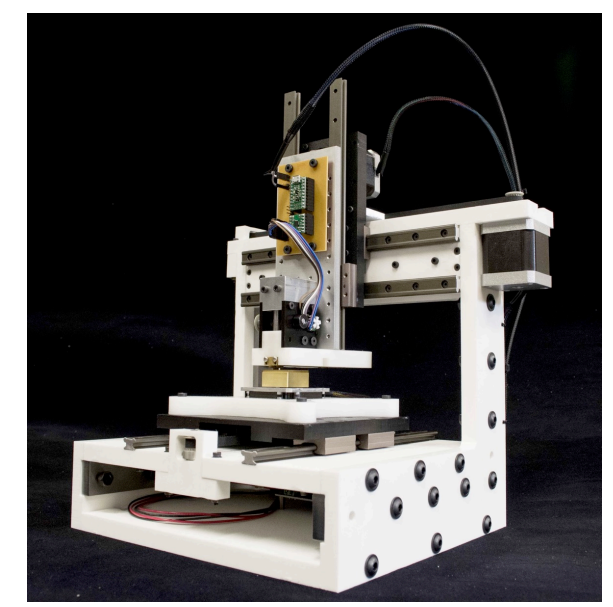

FIGURE 5 - ASSEMBLER MOTION GANTRY

\section{Stapler Mechanism}

The part placement mechanism must be fast, robust, and reliable. A number of potential mechanisms were considered and a worm-driven rack was ultimately selected for its compactness, small number of parts, and high gear ratio. This mechanism is pictured in Figure 6.
In the mechanism, a worm is driven by a DC gearmotor with a 100:1 gear reduction (from Pololu). The worm, in turn, drives a piston with an integrated rack. The piston tapers down to a blade which is just $0.25 \mathrm{~mm}$ thick (the thickness of a single building block). As the motor is driven, the piston is forced down, pushing out the next part in the magazine. Based on the torque of the DC gearmotor and the efficiency of the rackworm interface $(\sim 50 \%)$ the piston is expected to produce roughly $32 \mathrm{~N}$ of downward force for part-insertion, which is roughly double the expected $12 \mathrm{~N}$ of force needed to insert a part.

The motor is run with closed-loop feedback from a magnetic encoder attached to the pre-geared DC motor shaft and is current-limited to $0.5 \mathrm{~A}$ in software to ensure it does not overheat if stalled. The control algorithm is a basic PID controller, which drives the piston with a trapezoidal velocity profile between the two desired positions (up and down).

Using this mechanism and controller, we've measured the maximum part deposition rate at $0.72 \mathrm{~Hz}$. With additional tuning and component refinement this could be tuned to reach 1 $\mathrm{Hz}$.

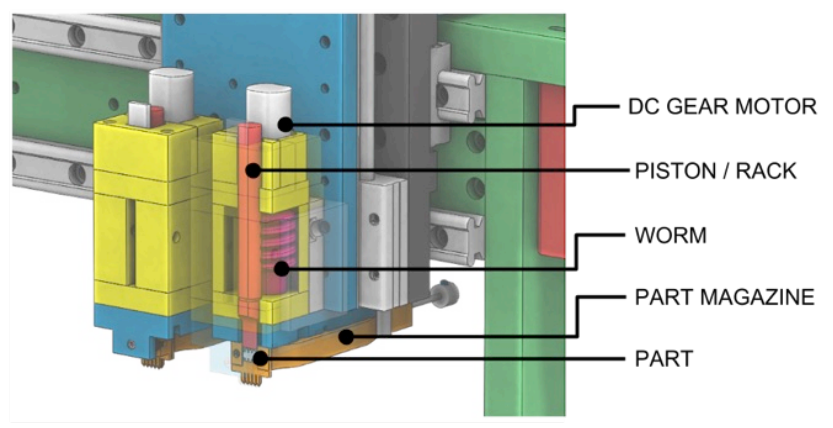

FIGURE 6 - STAPLER MECHANISM SECTION VIEW

\section{Stapler Magazine}

In order to enable parts to be refilled mid-build, the stapler magazine is decoupled from the stapler mechanism, allowing the magazine to be removed and reloaded easily. The magazine can hold up to 100 parts. A spring is used to preload the stack of parts against the front of the magazine with a pusher.

The most critical feature of this subsystem is the alignment mechanism to register the toolhead with the lattice. Alignment fingers on the front of the toolhead are used to correct for positioning errors prior to depositing a part. The pointed fingers reach into the negative space of the lattice to constrain the position in both $\mathrm{X}$ - and $\mathrm{Y}$-axes. The toolhead is made using a variety of fabrication processes including wire-EDM and manual machining.

The magazine is made to be easily removable from the rest of the toolhead. This is achieved through the use of a repeatable coupling mechanism. The tapered magazine is forced up against two dowel pins by a conical point setscrew, which interfaces with a conical hole on the front of the stapler 
magazine. This constrains all 6-degrees of freedom of the stapler magazine in such a way that it can be removed and reinserted midway through a build without any loss of precision. This mechanism is pictured in Figure 7.

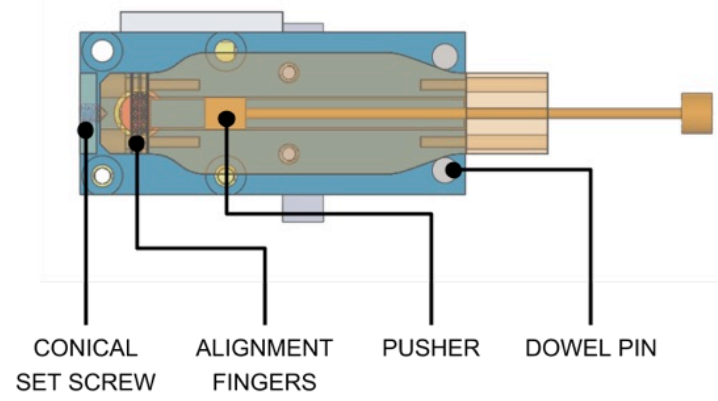

FIGURE 7 - STAPLER MAGAZINE FROM BELOW.

\section{CAD/CAM WORKFLOW}

As the size and complexity of discretely assembled structures increases, new software tools for Computer-aided Design (CAD) and Computer-aided Manufacturing (CAM) are needed. We have developed DMDesign, an end-to-end software workflow for the design, simulation, and toolpathing of discretely assembly of electronic structures. Figure 8 shows four screenshots of an LC structure designed from conductive and insulating parts in several stages of the DMDesign workflow.

Structures are designed from multiple materials in a hierarchical, 3D CAD interface. Within this interface, a user can toggle between an abstract, geometric representation (top left of Figure 8) and a realistic representation (top right of Figure 8) of the parts. Once a structure has been designed, DMDesign allows a user to assess the electronic and structural interconnectivity of the assembly, as well as simulate static, 3D potential fields in and around the assembly (bottom left Figure 8). Additionally, a user may export 3D meshes from their design to evaluate in other simulation packages.

Assembly designs are brought into the CAM workflow to plan and generate toolpathing and communicate in real time with the assembler. DMDesign communicates with the Stapler Assembler through a CAM protocol called G-Code. The GCode needed to assemble a given design is generated automatically by DMDesign, though users can gain low level control over the G-Code generation process via a scripting interface. Once generated, the G-Code may be simulated virtually to verify that the desired output is achieved (bottom right of Figure 8). Once verified, the G-Code is streamed to the assembler in real-time, and an open communication terminal allows users to take control of the process at any moment.

During the assembly process, the movements and state of the stapler assembler are mirrored by a virtual assembler within DMDesign. This platform was designed with the intention of eventually supporting more back and forth interaction between DMDesign, the assembler, and the partially-assembled structure; interesting applications include real-time sensing for increased part placement accuracy, intermediate calibration routines, and error detection and automatic correction.

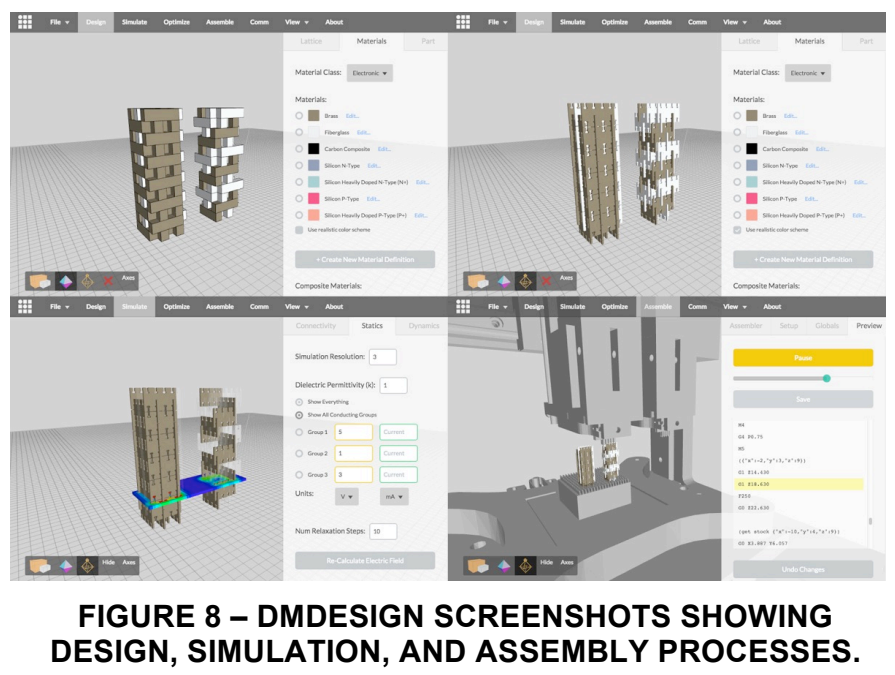

\section{EVALUATION}

\section{Joint Properties}

A critical part of this work is in understanding the mechanics at the interface between parts in order to ensure that they produce reliable and repeatable electrical contacts. The quality of the electrical contacts is crucial not only for reliable circuitry, but also for reducing parasitics and improving the quality of the assembled components. Here, we detail the measurement and evaluation of the electrical interfaces between parts.

To examine the performance of the joints within a threedimensional structure, we built a test specimen from purely conductive parts. The structure contains a total of 100 parts with five layers, each containing two rows of ten parts. Using an Instron material testing machine, we load the sample in compression and vary the load between $5 \mathrm{~N}$ and $40 \mathrm{~N}$, while measuring the conductance across the sample using a four-wire resistive measurement. Over 20 load cycles we see a ratcheting increase in the conductance of the sample from 120 Siemens to almost 220 Siemens, which can be seen in Figure 9. This increase in conductance indicates that the surface is being worn, flattening surface micropeaks, and increasing the effective contact area at the joint interfaces. 

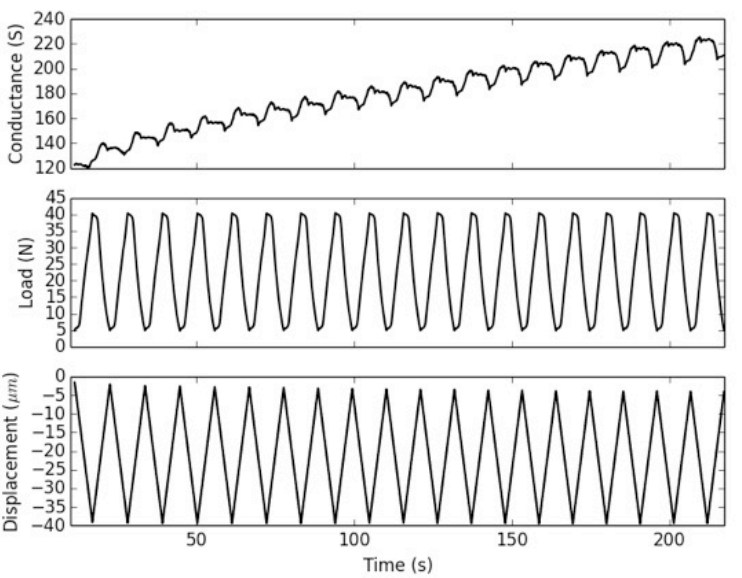

\section{FIGURE 9 - EFFECT OF LOAD CYCLING ON JOINT CONDUCTANCE.}

AS THE LOAD IS CYCLED, THE CONDUCTANCE MEASURED ACROSS THE STRUCTURE INCREASES FROM 120 SIEMENS TO 220 SIEMENS.

This flattening of surface micropeaks can be seen in comparing images taken with a scanning electron microscope of a joint before and after use. The before image (pictured on top in Figure 10) has much more surface roughness, especially on the joint side-wall, than the after image (pictured on the bottom of Figure 10).
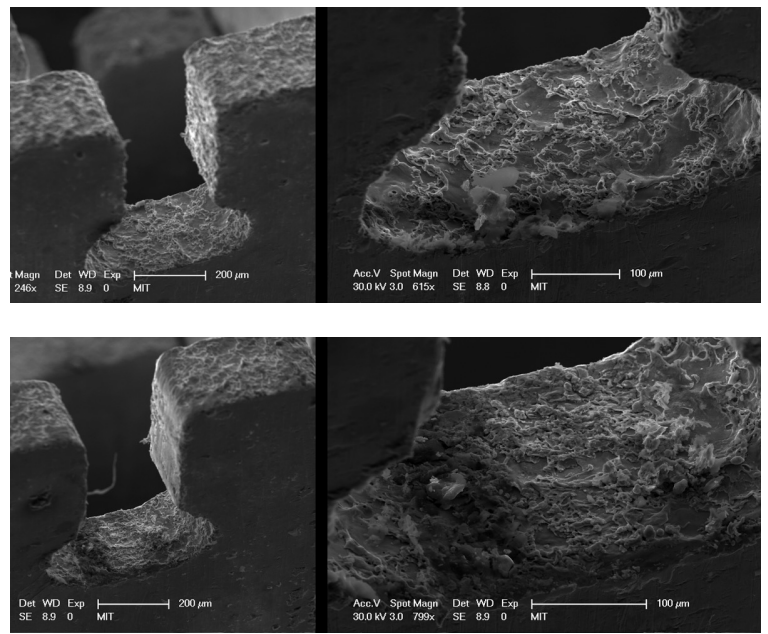

\section{FIGURE 10 - SCANNING ELECTRON MICROGRAPH SHOWING JOINT WEAR.}

THE UNUSED SLOT (TOP) APPEARS ROUGHER AND WITH MORE SURFACE ASPERITIES THAN DOES THE WORN SLOT (BOTTOM).

This testing indicates that in order to reduce the effect of load cycles on the conductivity of the joints, it is desirable to preload the assembled components in compression. By preloading, the microscopic sliding of the joint faces on each other is reduced and the conductance of the structure changes less with applied load.

\section{Machine Design}

The assembler is designed to exploit the digital material structure to correct positioning errors within a large margin. By using the alignment fingers at the bottom of the toolhead to register to the lattice for final positioning, the assembler should be able to correct for errors up to a theoretical maximum of $0.635 \mathrm{~mm}$ off in the $\mathrm{X}$ - or $\mathrm{Y}$-axes.

To test this, the machine was zeroed such that the fingers were perfectly centered in the negative space of the lattice. The machine was then commanded to move to deviations from this position and then lowered into the structure. We found that the machine was able to accommodate deviations of up to $0.5 \mathrm{~mm}$ in the X-and Y-axis. When the toolhead is $0.5 \mathrm{~mm}$ off of center, the tips of the alignment fingers sit just inside the negative space. More than $0.5 \mathrm{~mm}$ off of center, and the alignment fingers crash on the top flat surface of the top layer of parts in the structure. The maximum tolerable alignment error is illustrated in Figure 11.

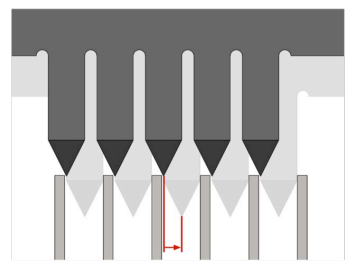

FIGURE 11 - ILLUSTRATION SHOWING THE POSITIONING ERROR TOLERANCE REGION. THE ALIGNMENT FINGERS ARE SHOWN AT THE LEFTMOST-END OF THEIR POSITIONING TOLERANCE REGION WITH RESPECT TO THE LATTICE.

The assembler should not only be error tolerant, but it should also be relatively fast at depositing parts and building volumes.

With default settings, a typical part placement speed for the assembler is approximately $0.2 \mathrm{~Hz}$ (or 1 part every 5 seconds). Given this, and the geometry of the parts and the lattice, we can calculate a volumetric build rate of $80 \mathrm{~mm}^{3} / \mathrm{min}$. This is on par with the build speed of commercial 3D printers, which take a few hours to build a one cubic inch structure.

Of course, with further tuning and testing, the same hardware could be used to assemble objects much faster, potentially up to $1 \mathrm{~Hz}$, at which point the stapler mechanism becomes the rate limiter.

\section{Capacitor Test Case}

To evaluate the entire workflow from design to characterization, we chose to design, assemble, and measure a small capacitor as a test case.

The capacitor is first designed in DMDesign and simulated using COMSOL. Both the conductive and insulating blocks are modeled in simulation to accurately account for the dielectric of the fiberglass. The simulation assumes the conductive blocks are perfect conductors and the fiberglass has a relative 
permittivity of 4.8 . Given these assumptions, the simulation predicts a capacitance of $4.85 \mathrm{pF}$ for the structure.

After designing and simulating the capacitor, an assembly sequence is generated and streamed to the assembler through DMDesign. The whole assembly process takes approximately 20 minutes, including refilling both magazines midway through. In this case we assembled two separate capacitors side by side, as seen in Figure 12.

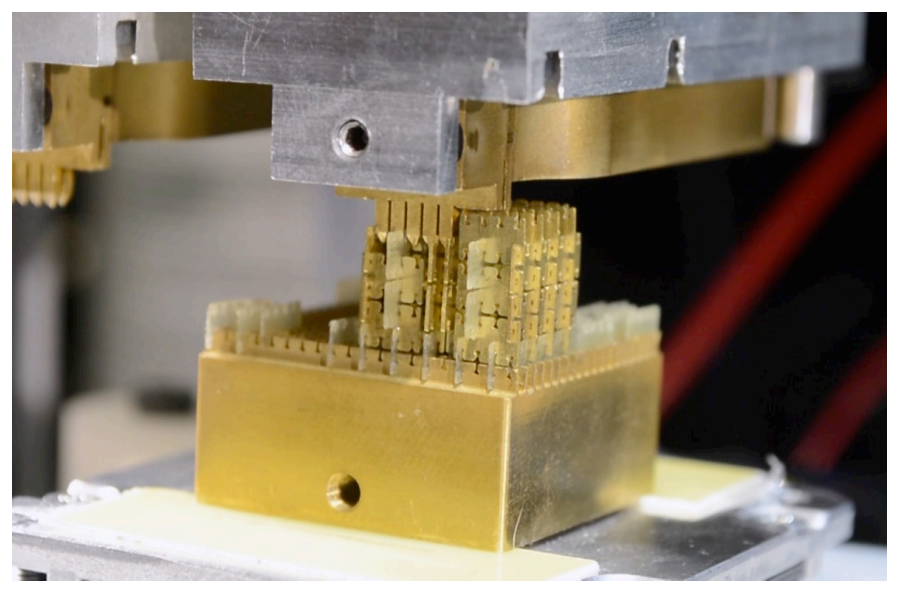

FIGURE 12 - TWO CAPACITORS BEING ASSEMBLED.

In total, three capacitors, which are shown in Figure 13, were measured using an LCR meter (HP 4623A) at $100 \mathrm{KHz}$ to determine how precisely they were assembled. The mean capacitance was measured to be $4.67 \mathrm{pF}$ with a standard deviation of $0.0044 \mathrm{pF}$, less than $1 \%$ of their mean value. This measurement falls well within $5 \%$ of the simulated capacitance without any form of calibration.

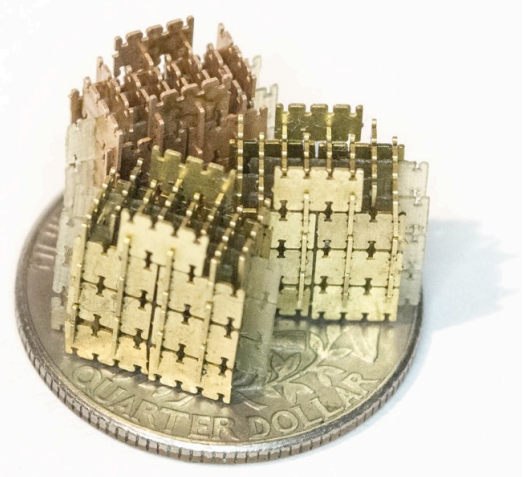

\section{FIGURE 13 - THREE ASSEMBLED CAPACITORS ON A QUARTER FOR SCALE.}

\section{DISCUSSION}

In order to compete with conventional electrical components, the size of the component parts needs to be scale down and the number of parts in the assemblies needs to be scale up. For example, to match the capacitive-density (Farads $/ \mathrm{mm}^{3}$ ) of a conventionally made $1 \mu \mathrm{F} 1206$ chip capacitor, the parts described in this work would need to be scaled down approximately three orders of magnitude, to parts $5 \mu \mathrm{m}$ in length.

Additionally, in order to scale this fabrication process to larger, more complex assemblies, a higher throughput assembly method is necessary. The assembly method described in this work may be sped up to part-deposition rates of approximately $1 \mathrm{~Hz}$ without significant hardware modifications. However, at this rate, an object composed of one billion parts would take almost 32 years to assemble (and would be approximately the size of a standard cubic pallet load).

Instead, we need a more parallel approach. Rather than devising a machine to place many parts at once (which makes it harder to detect and correct errors), we believe the way to speed up assembly is through the use of many distributed assemblers, each placing a single part at once. Through the use of error tolerance schemes similar to what we described in this work, these distributed assemblers could be made to be extremely robust and reliable.

\section{CONCLUSION}

We have demonstrated an end-to-end workflow for the discrete assembly of electronic digital materials. By using parts that structurally interlock with one another, we are able to assemble multi-material structures with highly conductive electrical pathways, enabling the fabrication functional electronic devices without the need for a binder or thermal annealing. We have developed an automated assembler that can programmatically assemble arbitrary structures from conductive and insulating block-types. Furthermore, by exploiting the structure of the digital materials, we have shown that the assembler can be made to be extremely error tolerant. Finally, we have demonstrated this end-to-end workflow through the design, simulation, and assembly of three capacitors, which were measured and confirmed to be functionally precise.

\section{ACKNOWLEDGMENTS}

This work was supported by NSF (CMMI-1344222), DARPA (W911NF-14-2-0063), NASA (NNX14AH75A), and CBA.

\section{REFERENCES}

[1] B. G. Compton and J. a. Lewis, "3D-Printing of Lightweight Cellular Composites," Adv. Mater., p. n/an/a, Jun. 2014.

[2] S. Shan, S. H. Kang, J. R. Raney, P. Wang, L. Fang, F. Candido, J. a. Lewis, and K. Bertoldi, "Multistable Architected Materials for Trapping Elastic Strain Energy," Adv. Mater., p. n/a-n/a, Jun. 2015.

[3] D. Raviv, W. Zhao, C. McKnelly, A. Papadopoulou, A. Kadambi, B. Shi, S. Hirsch, D. Dikovsky, M. Zyracki, 
C. Olguin, R. Raskar, and S. Tibbits, "Active Printed Materials for Complex Self-Evolving Deformations," Sci. Rep., vol. 4, p. 7422, 2014.

[4] A. Russo, B. Y. Ahn, J. J. Adams, E. B. Duoss, J. T. Bernhard, and J. A. Lewis, "Pen-on-Paper Flexible Electronics," Adv. Mater., vol. 23, no. 30, pp. 34263430, 2011.

[5] D. Zhao, T. Liu, J. G. Park, M. Zhang, J. M. Chen, and B. Wang, "Conductivity enhancement of aerosol-jet printed electronics by using silver nanoparticles ink with carbon nanotubes," Microelectron. Eng., vol. 96, pp. 71-75, 2012.

[6] "Voltera." [Online]. Available: http://voltera.io/. [Accessed: 26-Nov-2015].

[7] "Voxe18." [Online]. Available: http://www.voxel8.co/. [Accessed: 27-Nov-2015].

[8] K. Sun, T. S. Wei, B. Y. Ahn, J. Y. Seo, S. J. Dillon, and J. a. Lewis, "3D printing of interdigitated Li-ion microbattery architectures," Adv. Mater., vol. 25, no. 33, pp. 4539-4543, 2013.

[9] E. Malone, K. Rasa, D. Cohen, T. Isaacson, H. Lashley, and H. Lipson, "Freeform fabrication of zinc-air batteries and electromechanical assemblies," Rapid Prototyp. J., vol. 10, no. 1, pp. 58-69, 2004.

[10] J. J. Adams, E. B. Duoss, T. F. Malkowski, M. J. Motala, B. Y. Ahn, R. G. Nuzzo, J. T. Bernhard, and J. a Lewis, "Conformal printing of electrically small antennas on three-dimensional surfaces.," Adv. Mater., vol. 23, no. 11, pp. 1335-40, Mar. 2011.

[11] S. B. Fuller, E. J. Wilhelm, and J. M. Jacobson, "Ink-jet printed nanoparticle microelectromechanical systems," J. Microelectromechanical Syst., vol. 11, no. 1, pp. 5460, 2002.

[12] G. A. Popescu, T. Mahale, and N. A. Gershenfeld, "Digital materials for digital printing," NIP Digit. Fabr. ..., pp. 1-4, 2006.

[13] R. MacCurdy, a. McNicoll, and H. Lipson, "Bitblox: Printable digital materials for electromechanical machines," Int. J. Rob. Res., Jul. 2014.

[14] J. D. Hiller and H. Lipson, "Fully Recyclable MultiMaterial Printing," Solid Free. Fabr. Symp., pp. 98106, 2009.
[15] J. Hiller and H. Lipson, "Methods of Parallel Voxel Manipulation for 3D Digital Printing," in Proceedings of the 18th solid freeform fabrication symposium., 2007, p. 12.

[16] K. Gilpin, A. Knaian, and D. Rus, "Robot pebbles: One centimeter modules for programmable matter through self-disassembly," 2010 IEEE Int. Conf. Robot. Autom., pp. 2485-2492, May 2010. 
ANNEX A

\section{AUTOMATED DESKTOP PUNCH PRESS}
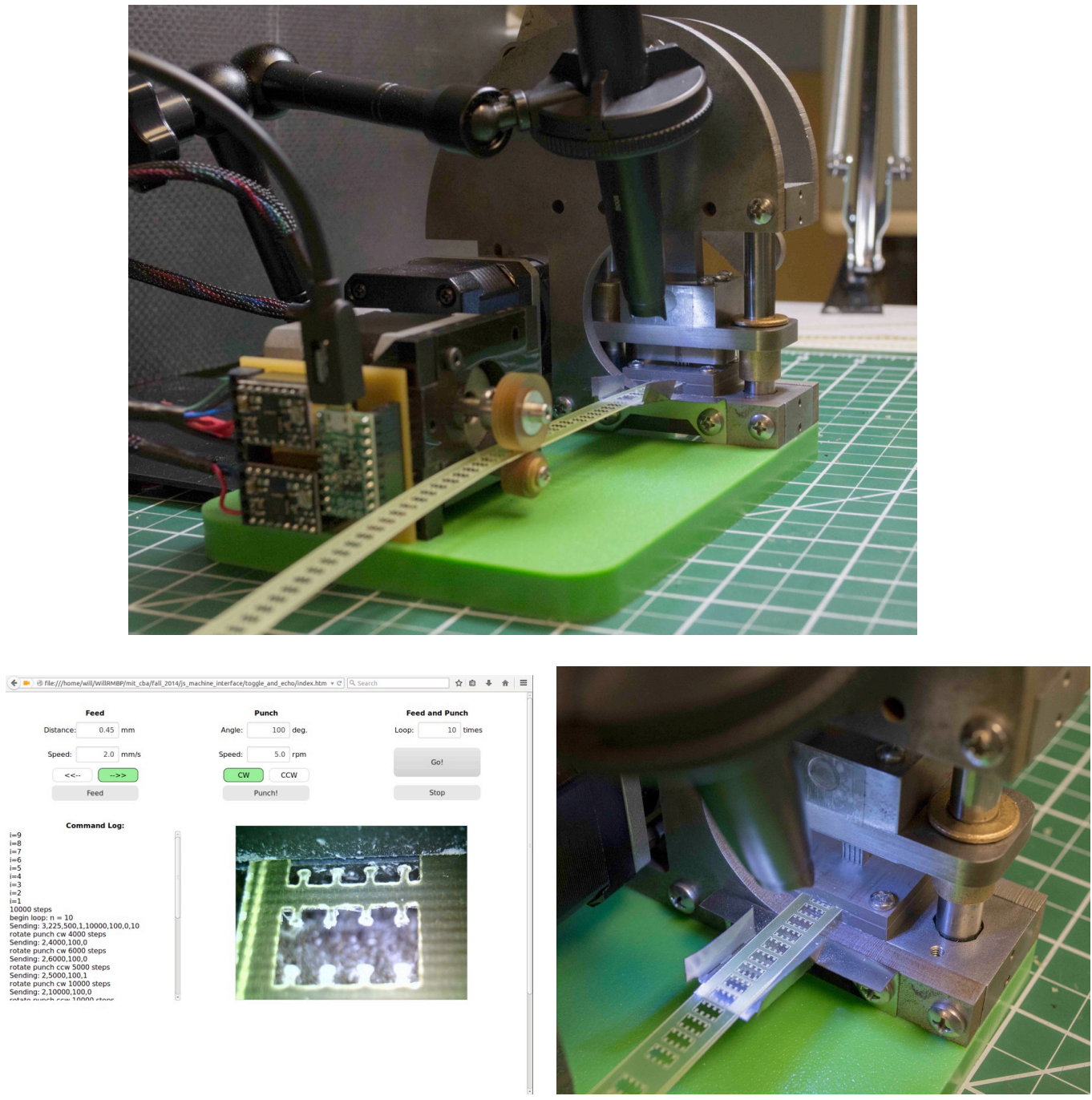

In order to produce the insulating parts in mass, we developed an automated desktop punchpress. The machine takes in a strip of the fiber-epoxy composite and stamps out individual parts, advancing the strip between each stamp. The machine is controlled through a javascript interface, enabling online verification of strip-advancement and measurement of tool and die wear. Using this simple machine, we are able to process raw material into digital material feedstock for the stapler assembler. 